\title{
Dissipation-free ion cooling in plasma flows with frozen-in fields
}

\author{
H.-J. Fahr and M. Siewert
}

\begin{abstract}
Argelander Institut für Astronomie der Universität Bonn, Abteilung für Astrophysik und Extraterrestrische Forschung, Auf dem Huegel 71, 53121 Bonn, Germany

e-mail: msiewert@astro.uni-bonn.de
\end{abstract}

Received 20 December 2007 / Accepted 1 April 2008

\section{ABSTRACT}

\begin{abstract}
We reinvestigate the pick-up ion phasespace transport in the inner heliosphere, this time neglecting stochastic processes like quasilinear wave-particle interactions. In the conventional literature on this subject, adiabatic deceleration of ions at their co-motion with the solar wind bulk flow has been considered, but, as demonstrated here, it is an inadequate concept in the region of the supersonically expanding solar wind. Instead, one has to consider the effect on the ion distribution function by ions at their co-motion with the solar wind having to conserve the two magnetic CGL-invariants. Conservation of the magnetic particle moment leads to velocity drifts in the velocity plane perpendicular to the magnetic field $\boldsymbol{B}$, while conservation of the second CGL invariant leads to corresponding velocity drifts along the magnetic field. As we can show, the conservation of the second CGL invariant is therefore a mere consequence of field-parallel dispersive particle motions in diverging plasma flows, such as the solar wind, flowing into regions with differential bulk motion. Taking both effects of velocity drifts together, one can then calculate the resulting ion distribution functions from an adequate Boltzmann-Vlasov transport equation and find that these functions are general power laws with ubiquitous power index of $\gamma_{\text {pui }}=-5$. The same power index is also found for pick-up ions at higher energies, where they are subject to stochastic energy diffusion processes by wave-particle interactions. This is perhaps the explanation of the unbroken pick-up ion power laws from the $1 \mathrm{keV}$ to the $100 \mathrm{keV}$ energy level.
\end{abstract}

Key words. Sun: solar wind - magnetic fields - Sun: magnetic fields - magnetohydrodynamics (MHD) - plasmas

\section{Introduction}

In an interplanetary magnetic field $\boldsymbol{B}(r)$, which is a function of the solar distance $r$, it is well-known that any freely convected, collision- and dissispation-free ion possesses a conserved magnetic moment $\mu=m v_{\perp}^{2} / 2 B$, where $m, v_{\perp}$, and $B$ denote the ion mass $m$ and the component of the ion velocity $\boldsymbol{v}$ perpendicular to the magnetic field $\boldsymbol{B}$. The gyro-averaged Lorentz force acting on these ions keeps $\mu$ constant, leading to a decrease in $v_{\perp}^{2}$ with decreasing magnetic field strength $B$. For a pitchangle-isotropic distribution function, i.e. for $\partial f / \partial \vartheta=0$, this can be interpreted as an induced convection in velocity space due to a drift (see e.g. Fahr \& Lay 2000) $\dot{v}_{m}=\sqrt{\frac{3}{2}}\left\langle\dot{v}_{\perp}\right\rangle_{\vartheta}$.

The representative Boltzmann-Vlasov equation (BVE) for pick-up ions in the rest frame of the sun (SF) is given under these conditions and in the stationary case by (Fahr 2007) as

$\left(\boldsymbol{U} \cdot \nabla_{r}\right) f+\left(\frac{\mathrm{d}}{\mathrm{d} t} \boldsymbol{v}_{m} \cdot \nabla_{v}\right) f=U \frac{\partial f}{\partial r}+U \frac{\partial v_{m}}{\partial r} \frac{\partial f}{\partial v}=P(r, v)$,

while the corresponding BVE in the (accelerated) rest frame of the solar wind (WF), describing the same system, takes the form

$\frac{\partial \tilde{f}}{\partial t}+\frac{1}{v^{2}} \frac{\partial}{\partial v}\left(v^{2} \dot{v}_{m} \tilde{f}\right)=\tilde{P}(t, v)$

Here, the second term on the left hand side describes the velocity-space divergence of the phasespace flow connected with the acceleration $\dot{v}_{m}=\mathrm{d} v_{m} / \mathrm{d} t$. The coordinate $t=t(r) \mathrm{de}-$ notes the proper time in the co-moving reference frame (WF). Furthermore, $\tilde{P}(r, v)$ is the local pick-up ion injection rate given by $\tilde{P}(r, v)=\beta(r) \frac{1}{4 \pi v^{2}} \delta(v-U)$, with $\beta(r)$ the local pick-up ion production rate. In contrast, in the SF, taking $\mathrm{H}$-atom velocities as negligibly small with respect to the wind velocity $U$, the corresponding injection term is given by $P(r, v)=\beta(r) \frac{1}{4 \pi v^{2}} \delta(v)$.

The acceleration term $\dot{v}$ appearing in these equations may now be derived using the conservation of the magnetic moment. For a pitchangle isotropic distribution function, the velocity magnitude $v$ shrinks by about the same rate as $v_{\perp}$ does, since

$\frac{\mathrm{d}}{\mathrm{d} t}\left\langle v_{\perp}\right\rangle_{\vartheta}=\left\langle\dot{v}_{\perp}\right\rangle_{\vartheta}=\frac{\mathrm{d}}{\mathrm{d} t}\left(2 \pi v \int_{0}^{\pi} \sin ^{2} \vartheta \mathrm{d} \vartheta\right)=\frac{\pi}{4} \dot{v}$

In the standard Parker model for the solar magnetic field (Parker 1958), the field is frozen-in into the solar wind, i.e. co-moving with the plasma and the pick-up particles. At greater distances $r$ from the sun (i.e. $r \geq 5 \mathrm{AU}$ ), the magnetic field is nearly azimuthal and decreases by $(1 / r)$. An isotropic distribution function will then be cooled by a drift

$\dot{v}_{m}=-U \frac{v}{r}$

which follows from the drift given at the end of the first paragraph and $\left\langle v_{\perp}^{2}\right\rangle_{\vartheta}=\frac{2}{3} v^{2}$. The radial velocity gradient associated with this process follows from $\mathrm{d} / \mathrm{d} t=U \mathrm{~d} / \mathrm{d} s$,

$\frac{\mathrm{d} v_{m}}{\mathrm{~d} r}=\frac{1}{U} \dot{v}_{m}=-\frac{v}{r}$.

As a reminder, for the more radial field at smaller distances, falling off with $r^{-2}$, one simply gets an additional factor 2 in the above relations. 
In the case of pure adiabatic deceleration, as considered by Vasyliunas \& Siscoe (1976), Isenberg (1987), Chalov \& Fahr (1996), Mall (2000), instead of the magnetically modified adiabatic deceleration value found in Eq. (4), one obtains the milder deceleration rate given by $\dot{v}_{m}=-\frac{2}{3} U v / r$, which would then lead to different ion energy spectra, as we show later in this paper.

\section{Collision-free magnetic cooling}

The cooling formalism introduced in the last section strongly relies on sufficiently rapid pitchangle isotropisation by quasilinear interactions of ions with MHD (Alfven-) waves (see Lee \& Ip 1987; Freund \& Wu 1988) and other dissipative processes (see Schlickeiser 1989), coupling the perpendicular velocity components to the parallel ones, and indirectly transforming an ansisotropic process, which acts only on the perpendicular velocity components, into an isotropic process acting on all velocity components. In the absence of these isotropisation processes, though, classical adiabatic cooling, as introduced above, leaves the velocities parallel to the magnetic field untouched, leading therefore to increasingly degenerated ion distribution functions with growing distance. As recognised much earlier (Griffel \& Davis 1969; Fahr \& Shizgal 1983), this would lead to significant complications with the radially expanding solar wind. Griffel \& Davis (1969) studied what collision rate on account of hypothetical, non-Coulomb-type processes would be required to reisotropise the ions, obtaining frequencies much higher than the Coulomb collision frequencies.

Interestingly enough, this well-known problem may be solved by considering not only the conservation of the magnetic moment (i.e. the first CGL invariant), but also the second CGL invariant. Formally, the first CGL invariant $\left(C G L_{1}=\left(P_{\perp} / B \rho\right)\right)$ may be transformed into the magnetic moment conservation for a single particle, $\mu=m v_{\perp}^{2} / 2 B^{2}$, by applying the substitution $p_{\perp} / \rho \rightarrow v_{\perp}^{2}$ (see Chew et al. 1956; Fahr et al. 1977). This suggests that a similar approach is also possible for the second CGL invariant, $C G L_{2}=\left(P_{\|} B^{2} / \rho^{3}\right)$, which would lead to the particle invariance $c_{2}=v_{\|}^{2} B^{2} / \rho^{2}$. As demonstrated by Fahr (2007), this cooling process takes the form

$\frac{\mathrm{d} v_{\|}}{\mathrm{d} r}=-\frac{v_{\|}}{r}$.

This is, however, precisely the same cooling rate as the one for the perpendicular velocity components, suggesting that, as long as the particle formulation of the second CGL invariant is conserved at the individual particle level, an initially isotropic distribution will remain isotropic, providing a good solution for the old puzzle of the low anisotropies of the solar wind.

At the particle level, this invariant is given by

$\frac{\mathrm{d}}{\mathrm{d} t} v_{\|}=v_{\|} \frac{\mathrm{d}}{\mathrm{d} t}(\ln \rho-\ln B)$,

which does not correspond to any classically conserved quantity. Therefore, although our formalism seemingly allows an old puzzle to be solved, it introduces a new puzzle, namely the question how a velocity component parallel to a magnetic field might be modified.

\section{Physics of the $C G L_{2}$ invariance on the particle level}

The one point that is absent in Fahr (2007) is the question about the physics behind the second per-particle CGL invariant. In this section, we demonstrate that an answer to this problem is surprisingly simple, at least under solar wind conditions. Imagine ions co-moving with the solar wind plasma bulk flow with a bulk velocity $\boldsymbol{U}$ and a frozen-in magnetic field $\boldsymbol{B}$. Then, ions with the velocity dispersion $v_{\|}$with respect to the bulk flow, within the unit of time, move along the magnetic field at a slightly different place where a slightly different bulk velocity $\boldsymbol{U}^{\prime}=\boldsymbol{U}+\mathrm{d} \boldsymbol{U}$ prevails. Therefore, on account of the modified bulk flow velocity $\boldsymbol{U}^{\prime}$ of the reference frame in which $v_{\|}$is considered, this ion is located in an accelerated reference frame; therefore, it is expecting a non-Newtonian acceleration, which may be expressed in the mass-independent form

$\frac{\mathrm{d} \boldsymbol{v}_{\|}}{\mathrm{d} t}=-v_{\|} \frac{1}{B}(\boldsymbol{B} \cdot \nabla) \boldsymbol{U}$

To further develop the above vector expression, we make use of the frozen-in field condition, valid in the solar wind, which is given by

$\frac{\mathrm{d} \boldsymbol{B}}{\mathrm{d} t}=0=\boldsymbol{\nabla} \times[\boldsymbol{U} \times \boldsymbol{B}]$

leading to the vector relation

$0=(\boldsymbol{B} \cdot \nabla) \boldsymbol{U}-\boldsymbol{B}(\nabla \cdot \boldsymbol{U})-(\boldsymbol{U} \cdot \nabla) \boldsymbol{B}$.

Recalling furthermore that, for the solar wind and the frozen-in magnetic field according to Parker (1958), we may assume that

$\boldsymbol{U}=\boldsymbol{U}_{r}=$ const

and

$\boldsymbol{B}=\boldsymbol{B}_{r}+\boldsymbol{B}_{\varphi}$.

Using these expressions, the divergence of the solar wind flow is given by

$(\nabla \cdot \boldsymbol{U})=\frac{1}{r^{2}} \frac{\partial}{\partial r}\left(r^{2} U_{r}\right)=\frac{2 U_{r}}{r}$

leading to

$(\boldsymbol{B} \cdot \nabla) \boldsymbol{U}=\boldsymbol{B} \frac{2 U_{r}}{r}+U_{r} \frac{\partial}{\partial r}\left(\boldsymbol{B}_{r}+\boldsymbol{B}_{\varphi}\right)$.

Now, with the Archimedian Parker field (Parker 1958), we obtain

$B_{r}=B_{r 0}\left(r_{0} / r\right)^{2}$

$B_{\varphi}=B_{r 0}\left(r_{0} / r\right)^{2} \frac{r \Omega_{\odot}}{U}$

and

$\tan \varphi=\frac{B_{\varphi}}{B_{r}}=\frac{r \Omega_{\odot}}{U}$,

where $\Omega_{\odot}$ denotes the angular rotation frequency of the sun. Then, using these equations, we finally obtain

$\frac{\partial}{\partial r}\left(\boldsymbol{B}_{r}\right)=-\frac{2 \boldsymbol{B}_{r}}{r}$

and

$\frac{\partial}{\partial r}\left(\boldsymbol{B}_{\varphi}\right)=-\frac{\boldsymbol{B}_{\varphi}}{r}$.

Introducing this into Eq. (14), we obtain

$(\boldsymbol{B} \cdot \nabla) \boldsymbol{U}=\boldsymbol{B} \frac{2 U_{r}}{r}+U_{r}\left[-\frac{2}{r} \boldsymbol{B}_{r}-\frac{1}{r} \boldsymbol{B}_{\varphi}\right]=\frac{U_{r}}{r} \boldsymbol{B}_{\varphi}$ 
which, using Eq. (8) delivers

$\frac{\mathrm{d} \boldsymbol{v}_{\|}}{\mathrm{d} t}=-v_{\|} \frac{1}{B}\left(\frac{U_{r}}{r} \cdot \boldsymbol{B}_{\varphi}\right)$.

In this equation, only the velocity component parallel to the local field $\boldsymbol{B}$ appears, reducing this expression to

$\frac{\mathrm{d} v_{\|}}{\mathrm{d} t}=-v_{\|} \frac{1}{B^{2}}\left(\frac{U_{r}}{r} \boldsymbol{B} \cdot \boldsymbol{B}_{\varphi}\right)=-v_{\|} \frac{U_{r}}{r} \sin ^{2} \varphi$

Using Eq. (17), it is clear now that, for greater solar distances, i.e. $r \geq 5 \mathrm{AU}$, the above formula simplifies to the form

$\frac{\mathrm{d} v_{\|}}{\mathrm{d} t}=-v_{\|} \frac{U_{r}}{r} \frac{1}{1+\left(\frac{U_{r}}{r \Omega_{\odot}}\right)^{2}}=-v_{\|} \frac{U_{r}}{r}$,

and thus is identical to Eq. (6).

\section{Magnetic drifts in view of energy conservation laws}

\subsection{The perpendicular velocity component}

In this section, we identify the physical nature of the magnetic cooling. We begin with the perpendicular velocity component, which is classically associated with the magnetic moment. We assume that the magnetic field is frozen-in into the wind system according to the Parker model, i.e. this field is not constant in the bulk frame, and the temporal change is given by

$\frac{\partial B}{\partial t}=-U_{r} \frac{\partial B}{\partial r}$.

This time-variable magnetic field, however, automatically leads to the presence of an electric vortex field, as it follows from Maxwells equations that

$\boldsymbol{\nabla} \times \boldsymbol{E}_{\text {ind }}=-\frac{1}{c} \frac{\partial \boldsymbol{B}}{\partial t}=\frac{U_{r}}{c} \frac{\partial \boldsymbol{B}}{\partial r}$.

Then, we multiply this expression by the oriented surface of a gyrating ion and, using the Stokes identity to integrate over this surface, we may derive the electric field acting on the single particle at a distance of $r_{\mathrm{g}}$ from the gyration centre,

$E_{\text {ind }}=-\frac{1}{c} \frac{\pi r_{\mathrm{g}}^{2} \frac{\mathrm{d}}{\mathrm{d} t} B}{2 \pi r_{\mathrm{g}}}=r_{\mathrm{g}} \frac{U_{r}}{2 c} \frac{\mathrm{d} B}{\mathrm{~d} r}$.

Now, using this form of the electric field acting on the gyrating particle, we may write the energy transfer between the perpendicular component of the particle and the field as

$$
-\frac{1}{2} \frac{\mathrm{d}}{\mathrm{d} t}\left(\frac{m v_{\perp}^{2}}{2}\right)=e E_{\mathrm{ind}} v_{\perp}=e\left(r_{\mathrm{g}} \frac{U_{r}}{2 c} \frac{\mathrm{d} B}{\mathrm{~d} r}\right) v_{\perp} .
$$

Here, we get an additional factor $1 / 2$ on the left side because $v_{\perp}$ describes two degrees of freedom. Then, introducing the gyroradius $r_{\mathrm{g}}=c m v_{\perp} / e B$, we simplify this expression to

$\frac{\mathrm{d}}{\mathrm{d} t} v_{\perp}=\frac{v_{\perp} U_{r}}{B} \frac{\mathrm{d} B}{\mathrm{~d} r}$

which leads to Eq. (5) using the quasi-azimuthal Parker field. Therefore, the physical process behind the conservation of the magnetic moment is the generation of a local electric field, against which the charged particle is perfoming work, leading to an exchange of energy.

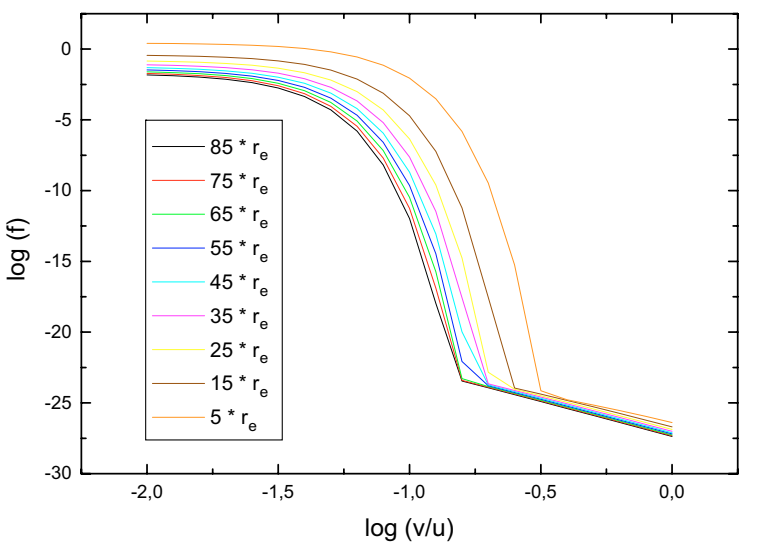

Fig. 1. Solar wind ions and pick-up ions compared at various distances from the sun. The thermal solar wind ions lead to the big plateau at low energies, while the pick-up ions give rise to the weaker high-energy power-law tail. The ordinate gives the ion velocity that has been normalised to the solar wind velocity $u=o\left(10^{5} \mathrm{~m} / \mathrm{s}\right)$, and the right end of the plotted velocity range corresponds to an energy of $1 \mathrm{keV}$. Solar wind temperatures are taken from Richardson et al. (2003), with $r_{e}=1 \mathrm{AU}$.

\subsection{The parallel velocity component}

While the identification of the perpendicular energy transfer as sketched here is an accepted and known fact following from classical electrodynamics, the parallel velocity decrease is less trivial. In a thermodynamical context, the expansion of a system is characterised by work performed by the system's internal pressure $p$. This process leads to a decrease in the pressure itself, and therefore, in a systematic reduction of velocities. Applied to an individual ion, the energy balance in this process is written in the form

$\frac{\partial}{\partial t}\left(\frac{m v_{\|}^{2}}{2}\right)=-\tilde{p}_{\|, I} \frac{\mathrm{d}}{\mathrm{d} t} \tilde{V}_{I}$,

where the subscript $I$ denotes the effective pressure and volume per single ion, i.e. $\tilde{p}_{\|, I}=\frac{m v_{\|}^{2}}{2 \tilde{V}_{I}}$ and $\tilde{V}_{i}=n^{-1}$, where $n$ is the particle number density. Inserting this into Eq. (29) and evaluating the derivative on the lefthand side, we obtain

$v_{\|} \frac{\mathrm{d}}{\mathrm{d} t} v_{\|}=\frac{v_{\|}^{2} U_{r} n}{2} \frac{\mathrm{d}}{\mathrm{d} r} n^{-1}$.

Now, we make use of the fact that, in a spherical symmetrical outflow such as the solar wind, the particle number density decreases as $n(r)=n_{0}\left(r_{0} / r\right)^{2}$, and we ultimately obtain

$\frac{\mathrm{d}}{\mathrm{d} t} v_{\|}=-v_{\|} \frac{U_{r}}{r}$

which is precisely the cooling process obtained from a straightforward conversion of the second CGL invariant (Fahr 2007).

\section{Solution of the Boltzmann-Vlasov equation for pick-up ions}

A solution for the BVE in the WF (see Eq. (2)) is given by Fahr (2007)

$\tilde{f}=\frac{1}{2 \pi} \frac{r \beta\left(\frac{v}{U} r\right)}{U} v^{-3}$ 
as can be easily verified by inserting this expression into Eq. (2) and multiplying by $v^{2}$. This distribution function can now be developed further for greater solar distances $r \geq r_{0}=5 \mathrm{AU}$ in the upwind hemisphere. At sufficiently large distances, the upwind $\mathrm{H}$-atom density may be considered essentially constant, which allows the assumption that $n_{H}\left(\frac{v}{U} r\right) \simeq n_{H}(r) \simeq n_{H, \infty}$. This is an acceptable approximation in the upwind hemisphere of the heliosphere for solar distances $r \geq 5$ AU and velocities $v \geq 0.2 U$. Then, one evidently finds the explicit form of the PUI distribution function by

$$
\tilde{f}=\frac{r}{2 \pi U} v_{\mathrm{ex}, E} r_{E}^{2}\left(\frac{v}{U} r\right)^{-2} n_{H, \infty} v^{-3}=\frac{v_{\mathrm{ex}, E} r_{E}^{2} U}{2 \pi r} n_{H, \infty} v^{-5},
$$

where the charge exchange frequency at $1 \mathrm{AU}$ is given by $\gamma_{\mathrm{ex}, E}=\sigma_{\mathrm{ex}}(n) n_{p, E} U$. Interestingly enough, this result means that, under pure magnetically modified adiabatic cooling as derived earlier in this paper, the resulting PUI distribution function is a power law with the power index $\alpha=-5$, which is precisely the same index as was found by Fisk \& Gloeckler (2006, 2007), using an entirely different approach, one based on the quasi-equilibrium state established between magnetoacoustically driven ion-energy diffusion and magnetoacoustic turbulence generation, i.e. for stochastic interaction between ions and MHD waves (see Chalov et al. 1997, 2003). Therefore, taking these two results together, the PUI distribution could perhaps be characterised by a thorough unbroken power law with the transitive uniform power index -5 both at high-velocity regions, where energy diffusion by wave-particle interaction dominates the form of the distribution function (Fisk \& Gloeckler 2007), and also at low-velocity regions, where the resonance condition breaks down and considerably different processes take over.

Another interesting question is whether the power-law spectrum at low energies is observable at all or whether this extension will be swallowed by the solar wind itself, which possesses a thermal distribution function. In Fig. 1, we present the combined distribution function of both the thermal and nonthermal components of the solar wind, which clearly demonstrates that the lower end of the PUI spectrum at $1 \mathrm{keV}$ should, in fact, be observable. Unfortunately, at greater solar distances, no corresponding data at energies below $5 \mathrm{keV}$ is available, although power laws with the predicted index of -5 have been observed above $30 \mathrm{keV}$ on the upstream and downstream sides of the solar wind termination shock (Decker et al. 2005).

At this point it is interesting to see that the conventionally applied adiabatic cooling process (Vasyliunas \& Siscoe 1976; Toptygin 1985; Mall 2000) leads to

$f_{\text {pui }}(r, v)=\frac{3 r}{8 \pi U^{4}} \beta\left(r_{v, \text { ad }}\right) \cdot\left(\frac{v}{U}\right)^{3 / 2}$

where $r_{v \text {,ad }}$ is given by $r_{v \text {,ad }}=r \cdot\left(\frac{v}{U}\right)^{3 / 2}$, which translates into the following form of the distribution function, when $\beta\left(r_{v, \text { ad }}\right)$ is expressed with the charge exchange ionisation frequency $v_{\mathrm{ex}, E}$ :

$f_{\text {pui }}(r, v)=\frac{3 v_{e x, E} r_{E}^{2}}{8 \pi r U^{4}} n_{H}\left(r_{v, a d}\right) \times\left(\frac{v}{U}\right)^{-3 / 2}$,

which, for larger distances where $n_{H}\left(r_{v, \text { ad }}\right) \simeq n_{H}(r) \simeq n_{H, \infty}$, has to be compared with the expression found for the modified cooling process derived here (see Eq. (33)).

\section{Conclusions}

We have studied the phasespace behaviour of solar wind ions and pick-up ions at their comotion with the solar wind bulk to greater interplanetary distances. As is generally known, ions have to conserve their magnetic moment, and when propagating with the solar wind bulk into regions with reduced magnetic field magnitude, they hence have to reduce their thermal velocities perpendicular to the local magnetic field $\boldsymbol{B}$. This type of modified adiabatic cooling is what all ions experience in the solar wind, so it represents an ion velocity drift in the component perpendicular to $\boldsymbol{B}$. In the absence of strong pitch-angle scattering, only the velocity components perpendicular to the magnetic field would be modified, leading to an increasingly anisotropic and unstable - plasma, which, in fact, is known to happen in the inner heliosphere close to the solar corona where the solar wind streams parallel to $\boldsymbol{B}$.

At greater solar distances, it is, in fact, possible to identify another mechanism, leading to the same cooling rate for the velocity components parallel to the magnetic field as the rate derived from the magnetic moment conservation. Taken together, these two processes prevent the solar wind from developing strong pressure anisotropies. This new effect is related to the differential motion of ions parallel to the local field $\boldsymbol{B}$. Since the solar wind density and pressure decrease with growing distance to the sun, these differential velocities decrease as well, leading to an effective loss of individual kinetic energies.

This effect of anisotropic adiabatic cooling of solar wind ions at greater solar distances leads to the emergence of power-law PUI spectra with a power-law index of -5 at low energies $(0.01-$ $1 \mathrm{keV}$ ), where resonant, dissipative processes do not dominate the system. Interestingly enough, this power law-index is also obtained at higher energies (Fisk \& Gloeckler 2006, 2007), under the assumption that magneto-acoustic wave-ion interaction leads to energy diffusion processes, allowing the spectrum to extend to much higher energies than those present directly after the injection into the solar wind. Taking this together with our current result, it follows that the power-law distribution function with a constant power index may dominate the solar wind over a much wider energy range than can be expected initially.

\section{References}

Chalov, S. V., \& Fahr, H. J. 1996, Sol. Phys., 168, 389

Chalov, S. V., Fahr, H.-J., \& Izmodeov, V. 1997, A\&A, 320, 659

Chalov, S. V., Fahr, H.-J., \& Izmodeov, V. 2003, J. Geophys. Res., 108(6), 1266

Chew, G. F., Goldberger, M. L., \& Low, F. E. 1956, Proc. R. Soc. London A, 236, 112

Decker, R. B., Krimigus, S. M., Roelof, E. C., et al. 2005, Science, 309, 2020

Fahr, H.-J. 2007, Ann. Geophys., 25, 1

Fahr, H.-J., \& Shizgal, B. 1983, Rev. Geophys. Space Phys., 21, 75

Fahr, H.-J., \& Lay, G. 2000, A\&A, 356, 327

Fahr, H. J., Bird, M. K., \& Ripken, H. W. 1977, A\&A, 58, 339

Fisk, L. A., \& Gloeckler, G. 2006, ApJ, 640, L79

Fisk, L. A., \& Gloeckler, G. 2007, PNAS, 104, 5749

Freund, H. P., \& Wu, C. S. 1988, J. Geophys. Res., 93, 14277

Griffel, D. H., \& Davis, L. 1969, Plan. Sp. Sc., 17, 1009

Isenberg, P. A. 1987, J. Geophys. Res., 92, 1067

Lee, M. A., \& Ip, W. H. 1987, J. Geophys. Res., 92, 11041

Mall, U. 2000, in The Outer Heliosphere: Beyond the Planets (Copernicus Society), 156

Parker, E. N. 1958, ApJ, 128, 664

Richardson, J. D., Wang, C., \& Burlaga, L. F. 2003, Geophys. Res. Lett., 30, 2207

Schlickeiser, R. 1989, ApJ, 336, 243

Toptygin, I. N. 1985, Cosmic rays in interplanetary magnetic fields, Geophysics and Astrophysics Monographs (Dordrecht: D. Reidel Publishing Company), 200

Vasyliunas, V. M., \& Siscoe, G. L. 1976, J. Geophys. Res., 81, 1247 